\title{
From Tsallis Scheme of high-energy pp collisions to Gen- eralized Multiplicity Distribution with Modified Combinants
}

\author{
M. Ghaffar ${ }^{1,3, *}, H . W$. Ang $^{1, * *}$, and A. H. Chan ${ }^{1, * * *}$ \\ ${ }^{1}$ Department of Physics, National University of Singapore, 2 Science Drive 3, Singapore 117542
}

\begin{abstract}
An analysis of the generalized multiplicity distribution, (GMD), has been made in high energy pp collisions. An attempt is made to reconcile the generating function of GMD with Tsallis statistics in order to find an interpretation of Tsallis parameter, $(q)$. The modified combinants, $\left(C_{j}\right.$ 's), for both GMD and NBD are obtained using the generating functions of these distributions. Our results show that single NBD does not lead to the oscillatory behaviour of the observed $C_{j}$. However, for GMD the corresponding $C_{j}$ not only oscillate but also show the fading-down feature of the experimentally observed $C_{j}$. This could help in future to analyse the physical process which is responsible for these oscillations.
\end{abstract}

\section{Introduction}

Long range interactions make standard statistical mechanics non-extensive. After the work of Bediaga et al. [1] and Beck [2], Tsallis statistics extension to hadronic collisions become in use as it gave the good fit for the hadronic productions in $e^{+} e^{-}$annihilation. We can cast the lowest order hard-scattering integral of transverse momentum spectra in high-energy $p p$ collisions in the Tsallis non-extensive form as [5].

$$
\left.\frac{d \sigma}{d y d \mathbf{p}_{T}}\right|_{y \sim 0}=\left.\frac{1}{2 \pi p_{T}} \frac{d \sigma}{d y d p_{T}}\right|_{y \sim 0}=A e_{q}^{-\frac{E_{T}}{T}},
$$

where

$$
e_{q}^{-\frac{E_{T}}{T}} \equiv\left[1-(1-q) \frac{E_{T}}{T}\right]^{\frac{1}{(1-q)}}
$$

Here $E_{T}=\sqrt{m^{2}+\mathbf{p}_{T}^{2}}$ and $m$ can be taken as pion mass $m_{\pi}$. The parameter $q$ is the Tsallis parameter and is related to the power index $n$ of the spectrum while the parameter $T$ is related to the average transverse momentum, $\left(p_{T}\right)$. The parameter $A$ is related to the multiplicity (per unit rapidity) after integration over $p_{T}$.

The Tsallis nonextensive distribution has been used for the description of transverse momenta of secondary particles produced in $p p$ collisions and gives excellent fits to the transverse momentum distributions by various collaborations at the LHC as shown in Fig. 1 [5].

\footnotetext{
*e-mail: mahumm_ghaffar@yahoo.com

**e-mail: ang.h.w@u.nus.edu

***e-mail: phycahp@nus.edu.sg
} 


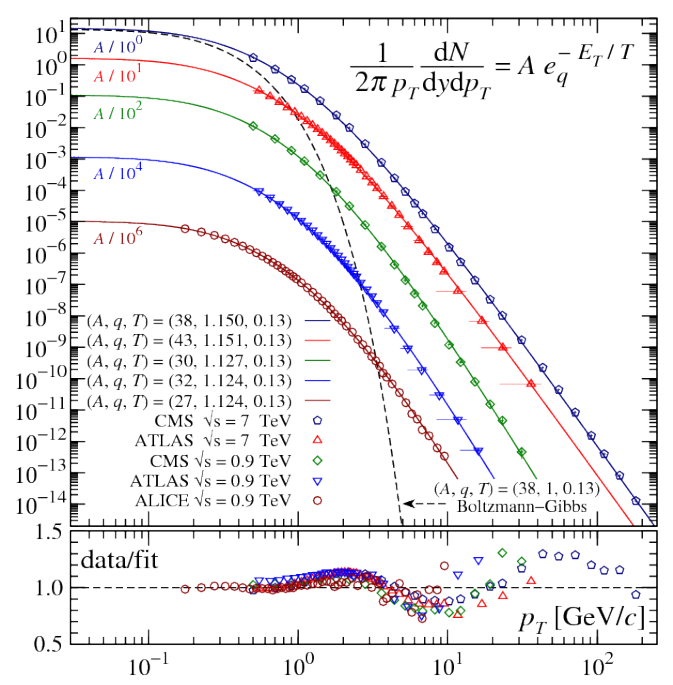

Figure 1. Comparison of Eq. (1) with the experimental transverse momentum distribution of hadrons in $p p$ collisions at central rapidity $y$. The corresponding Boltzmann-Gibbs distribution is shown as a dashed curve. The data/fit ratios are shown at the bottom where a log-periodic behaviour is observed on top of the $q$-exponential one [5].

\section{Connection between Tsallis approach and GMD}

Tsallis statistics was initially derived as a generalization of the Boltzmann-Gibbs statistics [3]. The application of Tsallis statistics to the study of multiparticle production was inspired by Aguiar and Kodama [4], where the generating function of the NBD was cast in the form reminiscent of that used by Tsallis. In a similar spirit, an attempt was made to cast the generating function of GMD in an attempt to reconcile it with Tsallis statistics, and to hypothesize on the physical interpretation of the $q$-parameter in this specific context.

GMD is derived from a parton branching model that describes the dynamics of quarkgluon production thus providing a more physical description of the events during collisions [6-8]. When $k^{\prime}$ parameter goes to zero, GMD reduces to NBD and this relation offers a parton branching description to NBD [8]. The probability distribution function of GMD and its corresponding generating function is given as:

$$
\begin{aligned}
P_{n}(G M D) & =\frac{\Gamma(n+k)}{\Gamma\left(n-k^{\prime}+1\right) \Gamma\left(k^{\prime}+k\right)}\left(\frac{\bar{n}-k^{\prime}}{\bar{n}+k}\right)^{n-k^{\prime}}\left(\frac{k+k^{\prime}}{\bar{n}+k}\right)^{k+k^{\prime}}, \\
f_{G M D}(s) & =\exp \left[\left(\bar{n}-k^{\prime}\right)(s-1)+\frac{\left(\bar{n}-k^{\prime}\right)^{2}}{2\left(k+k^{\prime}\right)}(s-1)^{2}+\ldots\right],
\end{aligned}
$$

where $\Gamma$ is the usual gamma function, $\bar{n}$ is the average number of particles, $k$ and $k^{\prime}$ corresponds to initial number of quarks and gluons respectively and $k+k^{\prime}=\frac{1}{q-1}$.

\subsection{Results for GMD fits at different centre of mass energies}

The graphs for GMD fits along with data to fit ratios are shown in Fig. 2a, 2b, 2c and 2d:

\section{Modified Combinants and comparison between NBD and GMD}

Modified combinants, $\left(C_{j}^{\prime} s\right)$ are defined in terms of generating functions of a given distribution i.e.,

$$
C_{j}=\left.\frac{1}{\langle N\rangle} \frac{1}{j !} \frac{d^{j+1} \ln G(z)}{d z^{j+1}}\right|_{z=0} .
$$




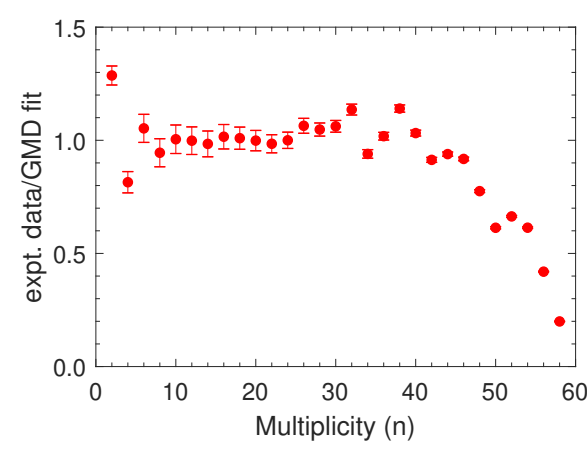

(a)

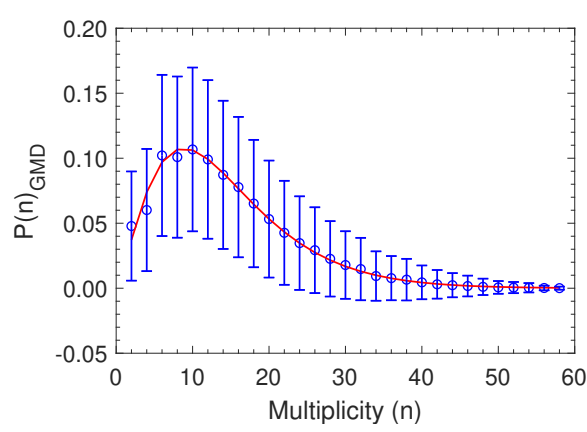

(c)

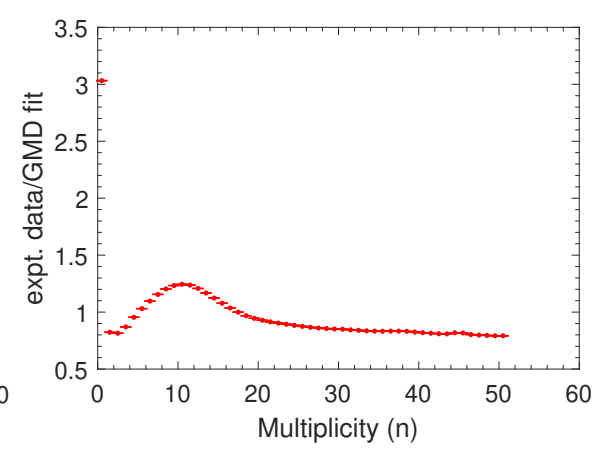

(b)

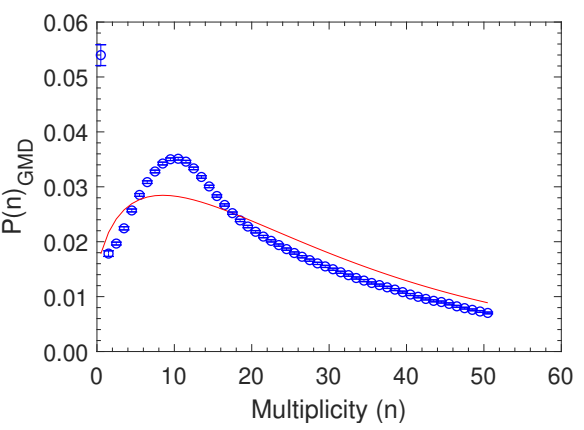

(d)

Figure 2. (a) Data to GMD fit ratio corresponding to multiplicity at $\sqrt{s}=0.2 \mathrm{TeV}$ with GMD parameters $k=1.67, k^{\prime}=1$ and $\bar{n}=14.36$. (b) Data to GMD fit ratio corresponding to multiplicity at $\sqrt{s}=7$ $\mathrm{TeV}$ with pseudorapidity range $|\eta|<2$ and GMD parameters $k=1.41, k^{\prime}=0$ and $\bar{n}=31$. (c) Comparison between data (circles) and GMD fit (line) at $\sqrt{s}=0.2 \mathrm{TeV}$ for $p \bar{p}$ collisions with GMD parameters $k=$ $1.67, k^{\prime}=1$ and $\bar{n}=14.36$. (d) Comparison between data (circles) and GMD fit (line) at $\sqrt{s}=7 \mathrm{TeV}$ and pseudorapidity range $|\eta|<2$ for $p p$ collisions with GMD parameters $k=1.41, k^{\prime}=0$ and $\bar{n}=31$.

By taking data to fit ratio, a wiggly structure as shown in Figs. $2 \mathrm{a}$ and $2 \mathrm{~b}$ is obtained suggesting some additional information hidden in $P(n)$ and $C_{j}^{\prime} s$ may provide a way to retrieve this information [9]. The generating functions $G(z)$ of NBD and GMD and their corresponding $C_{j}^{\prime} s$ are given as:

$$
\begin{gathered}
G(z)_{N B D}=\left(\frac{1-p}{1-z p}\right)^{k} \\
C_{j}(N B D)=\frac{k}{\langle N\rangle} p^{j+1} \\
G(t, z)_{G M D}=\frac{z^{k^{\prime}} e^{-\left(k+k^{\prime}\right) A t}}{\left[1-z\left(1-e^{-A t}\right)\right]^{\left(k+k^{\prime}\right)}},
\end{gathered}
$$

where $A$ is the average probability of parton branching process via gluon fission.

$$
C_{j}(G M D)=\frac{(-1)^{j+1}}{\langle N\rangle}\left[\frac{-k^{\prime}}{z^{j+1}}+\frac{\left(k^{\prime}+k\right)\left(k^{\prime}-\langle N\rangle\right)^{j+1}}{\left(k^{\prime} z+k-\langle N\rangle z+\langle N\rangle\right)^{j+1}}\right],
$$


where $z=\frac{1}{1+C}$ and $C$ is a constant of integration. The graphical representation of $C_{j}^{\prime} s$ of both NBD and GMD are shown in Fig. 3a and 3b:

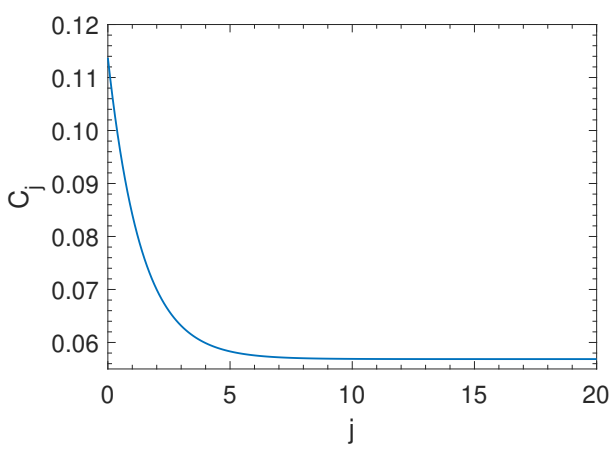

(a)

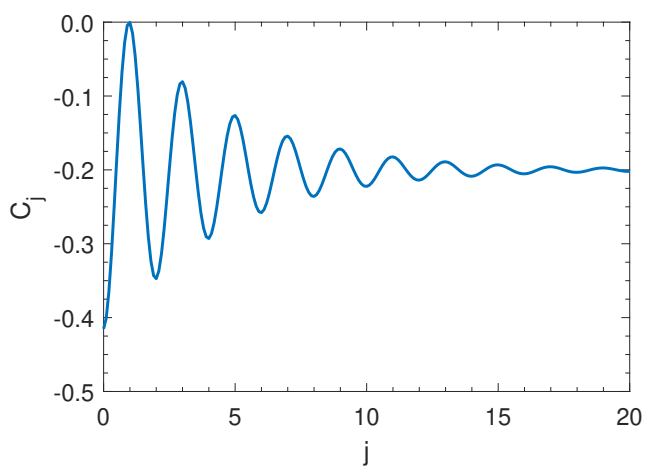

(b)

Figure 3. (a) $C_{j}$ plot for NBD at $\sqrt{s}=7 \mathrm{TeV}$ for $p p$ collisions by taking $p=0.48, \bar{n}=25.5$ and $k=$ 1.45. This plot is clearly non-oscillating.(b) $C_{j}$ plot for GMD at $\sqrt{s}=30.4 \mathrm{GeV}$ for $p p$ collisions by taking $z=3, \bar{n}=10.54, k=4.78$ and $k^{\prime}=1.69$. This plot clearly shows the oscillatory $C_{j}^{\prime} s$ of GMD.

\section{Remarks}

When GMD is cast in the same form as Tsallis statistics, we see that the $q$-parameter takes an inverse relationship with the sum of $k$ and $k^{\prime}$, interpreted as the average number of quarks and gluons respectively. Although it is unclear why this form of functional reltionship exists, this work narrows down on the parameters that $q$ is a function of.

GMD is a good alternative to NBD as it offers a parton branching description to NBD which otherwise remains as a statistical distribution equation. The combinants $\left(C_{j}^{\prime} s\right)$ of GMD show an oscillatory behaviour which appear to be dependent on $k^{\prime}$ parameter. The oscillatory $C_{j}^{\prime} s$ have been discussed and verified experimentally [9]. However using a single NBD does not allow us to get this oscillatory behaviour. In future the oscillatory $C_{j}^{\prime} s$ of GMD can be studied in more detail at different centre of mass energies. This would allow for a better quantitative research of the multiparticle production processes.

\section{Acknowledgement}

We would like to thank Prof. Grzegorz Wilk for his valuable discussion during 48th ISMD 2018. We are currently working to publish this work in a journal.

\section{References}

[1] I. Bediaga, E.M.F. Curado and J.M. Miranda, Physica A 286, 156 (2000).

[2] C. Beck, Physica A 286, 164 (2000).

[3] C. Tsallis, J. Stat. Phys. 52, 479 (1988).

[4] C. E. Aguiar and T. Kodama, Physica A 320, 371-386 (2003).

[5] C-Y Wong, G. Wilk, L. J. L. Cirto and C. Tsallis, Phys. Rev. D 91, 114027 (2015).

[6] C. K. Chew, D. Kiang and H. Zhou, Phys. Lett. B 186, 411 (1987).

[7] A. H. Chan and C. K. Chew, Phys. Rev. D 41, 851 (1990).

[8] A. Dewanto, A. H. Chan, C. H. Oh, R. Chan \& K. Sitaram, Eur. J. C 57, 515-523 (2008).

[9] G. Wilk and Z. Włodarczyk, 48, ISMD 2018, To appear EPJ Conference Proceedings (2019). 